\title{
Radial solutions of a semilinear elliptic problem*
}

\author{
M. A. Herrero and J. J. L. Velázquez \\ Departamento de Matemática Aplicada, Facultad de Matemáticas, Universidad \\ Complutense, 28040 Madrid, Spain
}

(MS received 29 March 1990. Revised MS received 25 October 1990)

\section{Synopsis}

We analyse the set of nonnegative, global, and radial solutions (radial solutions, for short) of the equation

$$
-\Delta u+u^{p}=f \quad \text { in } \mathbb{R}^{N}, \quad N \geqq 1,
$$

where $0<p<1$, and $f \in L_{\text {loc }}^{1}\left(\mathbb{R}^{N}\right)$ is a radial and almost everywhere nonnegative function. We show that radial solutions of (E) exist if $f(r)=o\left(r^{2 p / 1-p}\right)$ or if $f(r) \approx c r^{2 p / 1-p}$ as $r \rightarrow \infty$, where

$$
0<c<c_{*} \quad \text { and } \quad c_{*}=\left(p^{p / 1-p}-p^{1 / 1-p}\right)\left(\frac{(1-p)^{2}}{2(N(1-p)+2 p)}\right)^{p / 1-p}
$$

When $f(r)=c_{*} r^{2 p / 1-p}+h(r)$ with $h(r)=o\left(r^{2 p / 1-p}\right)$ as $r \rightarrow \infty$, radial solutions continue to exist if $h(r)$ is sufficiently small at infinity. Existence, however, breaks down if $h(r)>0$,

$$
h(r) \geqq \frac{r^{2 p / 1-p}}{(\ln r)^{\gamma}} \quad \text { as } \quad r \rightarrow \infty \text { with } 0<\gamma<2 .
$$

Whenever they exist, radial solutions are characterised in terms of their asymptotic behaviour as $r \rightarrow \infty$.

\section{Introduction and description of results}

Consider the semilinear elliptic equation

$$
-\Delta u+u^{p}=f \quad \text { with } f(x) \in L_{\mathrm{loc}}^{1}\left(\mathbb{R}^{N}\right), \quad N \geqq 1 .
$$

By a solution of (1.1), we shall mean a function $u \in L_{\text {loc }}^{1}\left(\mathbb{R}^{N}\right)$ such that (1.1) holds in $D^{\prime}\left(\mathbb{R}^{N}\right)$. When $p>1, \mathrm{H}$. Brézis has proved in [2] that (1.1) has a unique solution, irrespective of the behaviour of $f(x)$ when $|x| \rightarrow \infty$. This fact is in sharp contrast with the situation corresponding to the sublinear case. As a matter of fact, T. Gallouët and J. M. Morel showed in [3] that, if $f \geqq 0$ almost everywhere and

$$
0<p<1
$$

there exist constants $k_{1}$ and $k_{2}$ such that if equation (1.1) has a nonnegative solution, then

$$
\limsup _{R \rightarrow \infty} R^{-(N+(2 p / 1-p))} \int_{|x| \leqq R} f(x) d x<k_{2} ;
$$

* Partially supported by CICYT Grant PB86-0112-C0202 and EEC Contract SC1-0019-C. 
if

$$
\limsup _{R \rightarrow \infty} R^{-(N+(2 p / 1-p))} \int_{|x| \leqq R} f(x) d x<k_{1},
$$

then equation (1.1) has a nonnegative solution.

In general $k_{1} \leqq k_{2}$, but no precise estimate on these constants (which depend on $p$ and $N$ ) is known. Among other results, it was also shown in [3] that if $f \geqq 0$ almost everywhere and (1.1), (1.2) has a nonnegative solution, then this problem has a minimal solution $u$, in the sense that $0 \leqq u \leqq v$ almost everywhere for any other nonnegative solution $v$. We shall keep to this terminology henceforth.

The question of characterising the whole set of solutions of (1.1), (1.2) was left open in [3]. In this paper, we shall assume that $f(x) \equiv f(r) \geqq 0$ almost everywhere where $r=|x|$, and confine ourselves to the analysis of radial solutions of (1.1), (1.2) under such assumption. Specifically, our aim is to describe the solutions of the ODE

$$
-u^{\prime \prime}-\frac{N-1}{r} u^{\prime}+u^{p}=f(r), \quad r>0
$$

in terms of the asymptotic behaviour of $f(r)$ as $r \rightarrow \infty$. When $f \equiv 0$, such study has been performed by us in [4]. It has been shown there that the set of global nonnegative solutions (the solutions, for short, in what follows) in the homogeneous case consists of the trivial solution $u(r) \equiv 0$, and a monoparametric family of functions $u_{k}(r),-\infty<k<+\infty$, such that

$$
u_{k}(r)=c_{p, N} r^{2 / 1-p}+k r^{(2 / 1-p)-\alpha}+o\left(r^{(2 / 1-p)-\alpha}\right) \quad \text { as } \quad r \rightarrow \infty,
$$

where

$$
\begin{gathered}
\alpha=\frac{1}{2}\left(\beta_{p, N}-\left(\beta_{p, N}^{2}-4(1-p) c_{p, N}^{p-1}\right)^{\frac{1}{2}}\right), \\
\beta_{p, N}=\frac{4}{1-p}+N-2, \quad c_{p, N}=\left(\frac{(1-p)^{2}}{2(N(1-p)+2 p)}\right)^{1 / 1-p} .
\end{gathered}
$$

Here and henceforth, we shall freely use the customary asymptotic notations, $o($ ), $O(), \approx$, and «. Notice that, in the homogeneous case, nontrivial solutions are determined by the second term in their asymptotic expansion as $r \rightarrow \infty$.

We now proceed to discuss our results. To begin with, we consider the case where $f(r)$ is "small", namely $f(r)=o\left(r^{2 p / 1-p}\right)$ as $r \rightarrow \infty$, and introduce some notation. For $R>0$, let $\xi_{R}(r)$ be a smooth and nonnegative function such that $0 \leqq \xi_{R} \leqq 1$, and

$$
\xi_{R}(r)=0 \quad \text { if } \quad r<R, \quad \xi_{R}(r)=1 \quad \text { if } \quad r>R+1 .
$$

Let $w_{R}(r) \equiv w_{R}(r ; f)$ be the solution of

$$
\begin{aligned}
& -w^{\prime \prime}-\frac{N-1}{r} w^{\prime}+c_{p, N}^{p} r^{2 p / 1-p}\left[\left(1+c_{p, N}^{-1} r^{-(2 / 1-p)} w\right)^{p}-1\right] \xi_{R}(r) \\
& =\xi_{R}(r) f(r) \text { for } r>0, \\
& w(0)=w^{\prime}(0)=0 .
\end{aligned}
$$

It will be shown later (cf. Lemma 2.1) that a unique solution of (1.7) exists such 
that

$$
w_{R}(r)=o\left(r^{2 / 1-p}\right) \quad \text { as } \quad r \rightarrow \infty .
$$

Let us define now $\sigma(r)$ as the solution (if any) of

$$
\begin{aligned}
& -\sigma^{\prime \prime}-\frac{N-1}{r} \sigma^{\prime}+p\left(c_{p, N} r^{2 / 1-p}+w(r)\right)^{p-1} \xi_{R}(r) \sigma(r)=0 \text { if } \quad r>0, \\
& \sigma(0)=1 . \quad \sigma^{\prime}(0)=0 .
\end{aligned}
$$

We shall see in Lemma 2.3 that there exists a unique global solution of (1.9) such that, for any $\varepsilon>0$ satisfying $\varepsilon<(2 / 1-p)-\alpha(\alpha$ given in $(1.5 b))$ there holds

$$
r^{(2 / 1-p)-\alpha-\varepsilon} \ll \sigma(r) \ll r^{(2 / 1-p)-\alpha+\varepsilon} \quad \text { as } \quad r \rightarrow \infty .
$$

We then have

THEOREM 1.1. Assume that $f(r)=o\left(r^{2 p / 1-p}\right)$ as $r \rightarrow \infty$. Then there exists $R>0$ such that the set of solutions of (1.4), (1.2) consists in

$$
\text { a function } u(r)=o\left(r^{2 / 1-p}\right) \text { as } r \rightarrow \infty \text { (the minimal solution) }
$$

and a monoparametric family of functions $u(r ; k), k \in \mathbb{R}$, such that

$$
u(r ; k)=c_{p, N} r^{2 / 1-p}+w_{R}(r)+k \sigma(r)+o(\sigma(r)) \text { as } r \rightarrow \infty
$$

where $w_{R}(r)$ and $\sigma(r)$ are given respectively in (1.7), (1.9).

When additional information on $f(r)$ is available, formula (1.11) can be made more precise. For instance, if

$$
\int^{+\infty} t^{-(1+p / 1-p)} f(t) d t<+\infty,
$$

then $\sigma(r)=\mu r^{(2 / 1-p)-\alpha}+o\left(r^{(2 / 1-p)-\alpha}\right)$ as $r \rightarrow \infty$, for some real $\mu$ (cf. Proposition 2.13) Therefore, if, say, $f(r) \approx r^{(2 / 1-p)-\sigma}$ as $r \rightarrow \infty$ for some $\delta>0$, we obtain

$$
u(r ; k)=c_{p, N} r^{2 / 1-p}+w_{R}(r)+k r^{(2 / 1-p)-\alpha}+o\left(r^{(2 / 1-p)-\alpha}\right) \quad \text { as } \quad r \rightarrow \infty,
$$

(cf. (1.5)). On the other hand, we do not know how to compare $w_{R}(r)$ with $\sigma(r)$ in the general case. This can be done, however, when $f(r)$ has the particular form selected above: see Remark 2.2 below.

We next discuss the case where $f(r)=c r^{2 p / 1-p}+g(r)$, with $g(r)=o\left(r^{2 p / 1-p}\right)$ as $r \rightarrow \infty$. To begin with, when $g(r) \equiv 0$, one readily checks that if $0<c<c_{*}$, where

$$
c_{*}=\left(p^{p / 1-p}-p^{1 / 1-p}\right) c_{p, N}^{p}
$$

then (1.4), (1.2) has two nonnegative solutions, $u_{i}(r)=c_{i} r^{2 / 1-p}$, where for $i=1,2, c_{i}$ are the ordered roots $\left(c_{1} \leqq c_{2}\right)$ of

$$
\lambda^{p}-\lambda c_{p, N}^{p-1}=c \text {. }
$$

When $c=c_{*}$, these two roots coalesce into a single one, $\bar{c}=p^{1 / 1-p} c_{p, N}$. Note that the choice $f(r)=c r^{2 p / 1-p}, 0<c \leqq c_{*}$, corresponds to the behaviour described in (1.3) (with equality replacing the strict inequality there). The explicit solutions $u_{i}(r)$ will play an important role in describing the asymptotics of solutions in this case. 
To state our next result, we define $\bar{w}_{R}(r) \equiv \bar{w}_{R}(r ; g)$ (respectively $\bar{\sigma}(r)$ ) as the solution of (1.7) with $c_{p, N}$ and $f$ replaced by $c_{2}$ and $g$ (respectively the solution of (1.9) with $c_{p, N}$ and $w_{R}(r)$ replaced by $c_{2}$ and $\left.\bar{w}_{R}(r)\right)$. We then have

THEOREM 1.2. Let $f(r)=c r^{2 p / 1-p}+g(r)$ with $0<c<c_{*}$ and $g(r)=o\left(r^{2 p / 1-p}\right)$ as $r \rightarrow \infty$. Then there exists $R>0$ such that the set of solutions of (1.4), (1.2) is made out of

a function $\bar{u}(r)=c_{1} r^{2 / 1-p}+o\left(r^{2 / 1-p}\right)$ as $r \rightarrow \infty$, where $c_{1}$ is the smallest root of (1.14) (the minimal solution),

and a monoparametric family of functions $\bar{u}(r ; k), k \in \mathbb{R}$,

$$
\bar{u}(r ; k)=c_{2} r^{2 / 1-p}+\bar{w}(r)+k \bar{\sigma}(r)+o(\bar{\sigma}(r)) \quad \text { as } \quad r \rightarrow \infty .
$$

Notice the analogy between (1.11) and (1.16). Under some extra conditions on $g(r)$ more precise information about the asymptotics of $\bar{w}_{R}(r)$ and $\bar{\sigma}(r)$ can be obtained. We refer to Section 2 for more details, but we shall stress here a particular case. Set $g(r) \equiv 0$ in Theorem 1.2. Keeping to our previous notation, we then have $\bar{w}_{R}(r) \equiv 0$ and

$$
\begin{gathered}
\bar{u}(r) \equiv c_{1} r^{2 / 1-p} \quad \text { (the minimal solution), } \\
\bar{u}(r ; k)=c_{2} r^{2 / 1-p}+k r^{(2 / 1-p)-\beta}+o\left(r^{(2 / 1-p)-\beta}\right) \quad \text { as } \quad r \rightarrow \infty,
\end{gathered}
$$

where $\beta=\beta(p, N)$ is a constant satisfying $0<\beta<2 / 1-p$ (compare with Proposition 2.14).

We conclude with a study of the case $f(r) \approx c_{*} r^{2 p / 1-p}$ as $r \rightarrow \infty$. The interesting point here is that, while whenever $\left(f(r)-c_{*} r^{2 p / 1-p}\right)$ is small enough as $r \rightarrow \infty$ there is still a monoparametric family of solutions, it is possible to select $f(r)=c_{*} r^{2 p / 1-p}+h(r)$ with $h(r)=o\left(r^{2 p / 1-p}\right)$ as $r \rightarrow \infty$ such that no solution of (1.4), (1.2) exists. More precisely, for $R>0$, let us define $w_{R}^{*}(r)=w_{R}^{*}(r ; h)$ as the solution of (1.7) with $c_{p, N} r^{2 / 1-p}$ replaced by the function $u_{a}(r)$ given by

$$
u_{a}(r)=\bar{c} r^{2 / 1-p}+a_{1} \frac{r^{2 / 1-p}}{(\ln r)}+a_{2} \frac{r^{2 / 1-p} \ln (\ln r)}{(\ln r)^{2}},
$$

where $\bar{c}=p^{1 / 1-p} c_{p, N}$, and $a_{1}, a_{2}$ are some positive constants (cf. (3.6)). We then have

THEOREM 1.3. Assume that $f(r)=c_{*} r^{2 p / 1-p}+h(r)$. Then

(a) if for some $\varepsilon>0, h(r)=O\left(\frac{r^{2 p / i-p}}{(\ln r)^{2+\varepsilon}}\right)$ as $r \rightarrow \infty$, there exists $R>0$ such that the set of solutions of (1.4), (1.2) consists of a function

$$
u^{*}(r)=\bar{c} r^{2 / 1-p}+o\left(\frac{r^{2 / 1-p}}{\ln r}\right) \quad \text { as } \quad r \rightarrow \infty, \quad \text { where } \bar{c}=p^{1 / 1-p} c_{p, N}
$$

(the minimal solution), and a monoparametric family of functions $u^{*}(r ; k), k \in \mathbb{R}$, 
such that

$$
\begin{aligned}
u^{*}(r ; k)= & \bar{c} r^{2 / 1-p}+a_{1} \frac{r^{2 / 1-p}}{(\ln r)}+a_{2} \frac{r^{2 / 1-p} \ln (\ln r)}{(\ln r)^{2}}+k \frac{r^{2 / 1-p}}{(\ln r)^{2}} \\
& +w_{R}^{*}(r)+O\left(\frac{r^{2 / 1-p}}{(\ln r)^{3-\varepsilon}}\right) \text { as } r \rightarrow \infty, \text { for any } \varepsilon \in(0,1) .
\end{aligned}
$$

(b) Assume now that there exist $b>0, r_{0}>1$ and $\gamma \in(0,2)$ such that

$$
h(r)>0, \quad h(r) \geqq \frac{b r^{2 p / 1-p}}{(\ln r)^{\gamma}} \quad \text { if } \quad r \geqq r_{0} .
$$

Then there is no solution of (1.4), (1.2).

When $h(r) \equiv 0$, one also has $w_{R}^{*}(r) \equiv 0$ in $(1.18)$, and the mini nal solution $u^{*}(r)$ reduces to the explicit one, $u^{*}(r)=\bar{c} r^{2 / 1-p}$, cf. Proposition 3.2.

We shall prove Theorems 1.1 and 1.2 in Section 2 below. The proof of Theorem 1.3 will then make the content of Section 3.

\section{The proofs of Theorems 1.1 and 1.2}

2.1. The case $f(r)=o\left(r^{2 p / 1-p}\right)$ as $r \rightarrow \infty$

Take $R>0$ fixed, let $\xi_{R}(r)$ be as in (1.6), and consider the initial value problem (1.7). We then have

LemMA 2.1. Let $f(r)$ be such that $f(r)=o\left(r^{2 p / 1-p}\right)$ as $r \rightarrow \infty$. Then, for any $\varepsilon \in(0,1)$ there exists $R>0$ such that $(1.7)$ has a unique global solution $w_{R}(r)$ satisfying

$$
\begin{gathered}
0 \geqq w_{R}(r) \geqq-(1-\varepsilon) r^{2 / 1-p} \quad \text { for any } r>0, \\
w_{R}(r)=o\left(r^{2 / 1-p}\right) \quad \text { as } \quad r \rightarrow \infty .
\end{gathered}
$$

Proof. Local existence and uniqueness in some interval $I \equiv[0, \delta] \subset[0,+\infty)$ follows from standard arguments. To see that (2.1) holds on $I$, we consider the auxiliary function

$$
\bar{w}(r) \equiv \bar{w}(r ; \varepsilon)=-(1-\varepsilon) c_{p, N} r^{2 / 1-p}
$$

and the differential operator

$$
L(\theta)=-\theta^{\prime \prime}-\frac{N-1}{r} \theta^{\prime}+\xi_{R} c_{p, N}^{p} r^{2 p / 1-p}\left[\left(1+c_{p, N}^{-1} r^{-(2 / 1-p)_{\theta}}\right)^{p}-1\right] .
$$

We readily check that

$$
L(\bar{w})-\xi_{R} f \geqq\left(\varepsilon^{p}-\varepsilon\right) c_{p, N}^{p} r^{2 p / 1-p}-\xi_{R} f .
$$

Therefore, taking $R>0$ large enough (depending on $\varepsilon$ and $f$ ), it follows that

$$
L(\bar{w})-\xi_{R} f \geqq 0
$$


and since $0=w_{R}(r) \geqq \bar{w}(r)$ for $0<r<R$, we deduce by the maximum principle that $w_{R}(r) \leqq 0$ for any $r \in I$ and

$$
w_{R}(r) \geqq \bar{w}(r, \varepsilon) \text { for any } r \in I \text {. }
$$

Moreover, $w_{R}(r)$ can then be continued for any $r>0$, and (2.1) holds. As to (2.2), we first note that

$$
\text { there exists } L=\lim _{r \rightarrow \infty} r^{-(2 / 1-p)} w_{R}(r) \text { and } 0 \geqq L>-\infty .
$$

To get (2.4), we argue by contradiction. Assume now that

$$
\liminf _{r \rightarrow \infty} r^{-(2 / 1-p)} w_{R}(r)=L_{1}<L_{2}=\limsup _{r \rightarrow \infty} r^{-(2 / 1-p)} w_{R}(r) .
$$

We then take $z(r)=k r^{2 / 1-p}$ with $L_{1}<k<L_{2}$. Since $f(r)=o\left(r^{2 p / 1-p}\right)$ as $r \rightarrow \infty$, we readily see that we may select $k$ such that $\left(L z-\xi_{R} f\right)$ has a definite sign for $r>0$ large enough. Assume for instance that $L z-\xi_{R} f \geqq 0$ for $r \geqq R>0$. By (2.5), there exists an interval $I=\left(R_{1}, R_{2}\right)$ with $R \leqq R_{1} \leqq R_{2}$ where $w_{R}(r)>z(r)$ and $w_{R}(r)=z(r)$ for $r=R_{1}, R_{2}$, which is a contradiction to the maximum principle.

We next claim that $L=0$. To see this, we use (1.7) and (2.4) to obtain for any $\delta>0$ sufficiently small

$$
w_{R}^{\prime \prime}+\frac{N-1}{r} w_{R}^{\prime} \geqq c_{p, N}^{p} r^{2 p / 1-p}\left[\left(1+c_{p, N}^{-1}(L-\delta)\right)^{p}-1\right]-f(r) .
$$

Integrating this inequality twice in $r$, one has that for large enough $r$

$$
w_{R}(r) \geqq c_{p, N} r^{2 / 1-p}\left[\left(1+c_{p, N}^{-1}(L-\delta)\right)^{p}-1\right]+o\left(r^{2 / 1-p}\right) .
$$

Therefore

$$
L=\lim _{r \rightarrow \infty} r^{-(2 / 1-p)} w_{R}(r) \geqq c_{p, N}\left[\left(1+c_{p, N}^{-1}(L-\delta)\right)^{p}-1\right] .
$$

We now let $\delta \downarrow 0$ in (2.6) to get

$$
1+\frac{L}{c_{p, N}} \geqq\left(1+\frac{L}{c_{p, N}}\right)^{p}
$$

which implies $L \geqq 0$, so that we finally have $L=0$ and (2.2) holds.

Remark 2.2. More detailed information about the asymptotics of $w_{R}(r)$ as $r \rightarrow \infty$ can be obtained if further specifications on $f(r)$ are known. For instance, if $f(r) \approx r^{(2 p / 1-p)-\delta}$ as $r \rightarrow \infty$ for some $\delta \in(0,2 p / 1-p)$, linearisation at infinity in (1.7) yields

$$
-w^{\prime \prime}-\frac{N-1}{r} w^{\prime}+p c_{p, N}^{p-1} r^{-2} w \approx r^{(2 p / 1-p)-\sigma}
$$

whence, setting $r=e^{y}$ and arguing as in [1, Chapter 2], we get

(i) if $\delta>\alpha$ then $w_{R}(r) \approx \theta_{1} r^{(2 / 1-p)-\alpha}$ as $r \rightarrow \infty$;

(ii) if $\delta<\alpha$ then $w_{R}(r) \approx \theta_{2} r^{(2 / 1-p)-\delta}$ as $r \rightarrow \infty$, for some real constants $\theta_{1}$ and $\theta_{2}$. 
For $R>0$ fixed and large enough, we now turn our attention to the problem

$$
\begin{gathered}
-\sigma^{\prime \prime}(r)-\frac{N-1}{r} \sigma^{\prime}(r)+p \sigma(r) \xi_{R}(r)\left(c_{p, N} r^{2 / 1-p}+w_{R}(r)\right)^{p-1}=0 \quad \text { if } \quad r>0, \\
\sigma(0)=1, \quad \sigma^{\prime}(0)=0 .
\end{gathered}
$$

There holds

Lemma 2.3. There exists a unique global solution $\sigma(r)$ of (2.7). Moreover, we have:

$$
\sigma(r)>0 \quad \text { for } \quad r>0, \quad \sigma(r) \rightarrow \infty \quad \text { as } \quad r \rightarrow \infty ;
$$

Let $\alpha$ be given in $(1.5 b)$; then for any $\varepsilon>0$ small enough,

$$
r^{(2 / 1-p)-\alpha-\varepsilon} \ll \sigma(r) \ll r^{(2 / 1-p)-\alpha+\varepsilon} \text { as } r \rightarrow \infty .
$$

Proof. Existence and uniqueness of local solutions is straightforward. By (2.2), the quantity in the braces in (2.7a) stays away from zero, and the solution is global. Moreover, after making the substitution $r=e^{y}$, we deduce from (2.2) and the results in [1, Chapter 2, Theorem 7] that

$$
\sigma(r)=k_{1} \psi_{+}(r)+k_{2} \psi_{-}(r)
$$

where

$$
\begin{aligned}
& r^{(2 / 1-p)-\left|\alpha_{+}\right|-\varepsilon} \ll \psi_{+}(r) \ll r^{(2 / 1-p)-\left|\alpha_{+}\right|+\varepsilon} \text { as } r \rightarrow \infty \text {, } \\
& r^{(2 / 1-p)-\left|\alpha_{-}\right|-\varepsilon} \ll \psi_{-}(r) \ll r^{(2 / 1-p)-\left|\alpha_{-}\right|+\varepsilon} \text { as } r \rightarrow \infty \text {, }
\end{aligned}
$$

and

$$
\alpha_{ \pm}=\frac{1}{2}\left(-\beta_{p, N} \pm\left(\beta_{p, N}^{2}-4(1-p) c_{p, N}^{p-1}\right)^{\frac{1}{2}}\right)
$$

so that (cf. (1.5))

$$
\left|\alpha_{+}\right|=\alpha, \quad \alpha_{-}<\alpha_{+}<0, \quad \frac{2}{1-p}-\left|\alpha_{+}\right|>0 \quad \text { and } \frac{2}{1-p}-\left|\alpha_{-}\right|<0,
$$

$\varepsilon>0$ being as in the statement of the Lemma. Suppose now that $k_{1}=0$. Then $\sigma(r) \rightarrow 0$ as $r \rightarrow \infty$. Moreover, multiplying by ( $\operatorname{sgn} \sigma)$ in (2.7a), and using Kato's inequality,

$\Delta|v| \geqq \Delta v \cdot \operatorname{sgn} v \quad$ in $\quad D^{\prime}\left(\mathbb{R}^{N}\right) \quad$ for $v \in L_{\mathrm{loc}}^{1}\left(\mathbb{R}^{N}\right)$ with $\quad \Delta v \in L_{\mathrm{loc}}^{1}\left(\mathbb{R}^{N}\right)$,

it follows that $|\sigma|$ is subharmonic, so that it cannot achieve a maximum at the interior of any ball. This gives a contradiction, since $\sigma(0)=1$ by assumption. Using again the maximum principle for subharmonic functions, we note that $\sigma$ cannot change sign, and since $\sigma(0)=1$, then $\sigma(r)>0$ for any $r$.

Proceeding further with the proof of Theorem 1.1, we set

$$
v(r)=d r^{(2 / 1-p)-\mid \alpha_{+}-\varepsilon},
$$


where $\varepsilon>0$ and $d>0$ are selected so that $2 / 1-p-\left|\alpha_{+}\right|-\varepsilon>0$ and

$$
-v^{\prime \prime}-\frac{N-1}{r} v^{\prime}+\frac{p c_{p, N}^{p-1} v}{r^{2}}=r^{(2 p / 1-p)-|\alpha+|-\varepsilon} .
$$

We now have

Lemma 2.4. Let $k$ be a fixed real number, and let $R>0$ be as in Lemma 2.1. Define now

$$
\varphi(r) \equiv \varphi(r ; k, R)=c_{p, N} r^{2 / 1-p}+w_{R}(r)+k \sigma(r)-v(r),
$$

where $w_{R}(r), \sigma(r)$ and $v(r)$ are respectively given in Lemmata 2.1, 2.3 and (2.12). Then there exists $M>0$ such that $(\varphi-M)_{+}$is a subsolution for $(1.4)$ in $\mathbb{R}^{N}$, i.e.

$$
-\Delta(\varphi-M)_{+}+(\varphi-M)_{+}^{p} \leqq f \text { in } D^{\prime}\left(\mathbb{R}^{N}\right) .
$$

Proof. For simplicity, we shall write $\Delta \varphi$ instead of $\varphi^{\prime \prime}+(N-1 / r) \varphi^{\prime}$. For large enough $r>0$, we compute

$$
\begin{aligned}
-\Delta \varphi+\varphi^{p}= & -c_{p, N}^{p} r^{2 p / 1-p}-\Delta w_{R}-k \Delta \sigma+\Delta v \\
& +\left(c_{p, N} r^{2 / 1-p}+w_{R}\right)^{p}\left(1+p\left(c_{p, N} r^{2 / 1-p}+w_{R}\right)^{-1}(k \sigma-v)\right) \\
& +O\left(\left(c_{p, N} r^{2 / 1-p}+w_{R}\right)^{p-2}\left(|\sigma|^{2}+|v|^{2}\right)\right) \\
= & f(r)-\left[-\Delta v+\frac{p v(r)}{c_{p, N}^{1-p} r^{2}}\left(1+p c_{p, N}^{-1} r^{-(2 / 1-p)} w_{R}\right)\right] \\
& +O\left(\left(c_{p, N} r^{2 / 1-p}+w_{R}\right)^{p-2}\left(|\sigma|^{2}+|v|^{2}\right)\right) \\
= & f(r)-r^{(2 p / 1-p)-\left|\alpha_{+}\right|-\varepsilon}+o\left(r^{(2 p / 1-p)-\mid \alpha_{+}-\varepsilon}\right) \\
& +O\left(r^{(2 p / 1-p)-2 \mid \alpha_{+}+2 \varepsilon}\right)+O\left(r^{(2 p / 1-p)-2 \mid \alpha_{+}+2 \varepsilon}\right)
\end{aligned}
$$

and since $\left|\alpha_{+}\right| \geqq 1$, we obtain that for some $R>0$ large enough

$$
-\Delta \varphi+\varphi^{p} \leqq f \text { if } r>R .
$$

We now select $M=M(R)$ such that $-\Delta(\varphi-M)_{+}+(\varphi-M)_{+}^{p} \leqq f$ in $D^{\prime}\left(\mathbb{R}^{N}\right)$ for $r \leqq R$, and the result follows.

Define now

$$
\lambda(r)=\left(E_{N} * f_{1}\right)(r),
$$

where $E_{N}$ is the fundamental solution of the laplacian in $\mathbb{R}^{N}$ and $f=\aleph_{B_{R}(0)} f+$ $\left(1-\aleph_{B_{R}(0)}\right) f \equiv f_{1}+f_{2}$. Here $\aleph_{B_{R}(0)}$ is the characteristic function of the ball $B_{R}(0)$, and $R>0$ is large enough so that $\left(1-\aleph_{B_{R}(0)}\right) f \in L_{\mathrm{loc}}^{\infty}\left(\mathbb{R}^{N}\right)$. Note that $\lambda(r) \geqq 0$ if $N \geqq 3$. A slight modification of the proof in Lemma 2.4 yields

Lemma 2.5. Assume that $N \geqq 3$. Let $k$ be a fixed real number, and let $R>0$ be as in Lemma 2.1. Define now

$$
\psi(r)=c_{p, N} r^{2 / 1-p}+w_{R}(r)+k \sigma(r)+\lambda(r)+v(r),
$$

where $w_{R}(r), \sigma(r)$ and $\lambda(r)$ are respectively given in (2.1), (2.7) and (2.14). Then there exists $M>0$ such that $(\psi+M)$ is a supersolution for $(1.4)$ in $\mathbb{R}^{N}$, i.e.

$$
-\Delta(\psi+M)+(\psi+M)^{p} \geqq f \text { almost everywhere in } \mathbb{R}^{N}
$$


Remark 2.6. When $N=1,2$, we adapt the previous argument as follows. Take $V_{0}(r)=1$ if $R_{1}<r<R_{1}+1$. $V_{0}(r)=0$ otherwise, where $R_{1}>0$ is so large that $f \in L_{\mathrm{loc}}^{\infty}$ for $r>R_{1} / 2$. Consider now the problem:

$$
-\Delta \lambda_{1}+V(r) \lambda_{1}=f_{1} \quad \text { in } \quad \mathbb{R}^{N}, \quad \lambda_{1} \geqq 0 \quad N=1,2 .
$$

Then (E) has a solution $\lambda_{1}(r)$. Moreover, by the results of [5], there exists $C>0$ such that $\lambda_{1}(r) \leqq C(1+\log r)$ if $N=2$. An explicit computation also shows that $\lambda_{1}(r) \leqq C(1+r)$ if $N=1$. We then replace $\lambda(r)$ in $(2.14)$ by $\lambda_{1}(r)$ to obtain that the result in Lemma 2.5 also holds true in this case.

Using Lemmata 2.4 and 2.5, as well as Remark 2.6 for the case $N \leqq 2$, standard techniques yield

Corollary 2.7. Assume that $f(r)=o\left(r^{2 p / 1-p}\right)$ as $r \rightarrow \infty$. Then there exists a solution $u(r ; k)$ of $(1.4)$ such that

$$
u(r ; k)=c_{p, N} r^{2 / 1-p}+w_{R}(r)+k \sigma(r)+o(\sigma(r)) \text { as } r \rightarrow \infty .
$$

It remains to show yet that, under our current assumptions, every solution of (1.4) behaves as in (2.15) or is the minimal solution $u_{0}(r)$ such that $u_{0}(r)=$ $o\left(r^{2 / 1-p}\right)$ as $r \rightarrow \infty$. To this end, we shall need the following technical result:

Lemma 2.8. Let $u(r)$ be a solution of

$$
-u^{\prime \prime}-\frac{N-1}{r} u^{\prime}+u^{p}=f(r), \quad f(r)=o\left(r^{2 / 1-p}\right) \quad \text { as } \quad r \rightarrow \infty .
$$

Then

(a) there exists $v>0$ such that

$$
u(r) \leqq c_{p, N} r^{2 / 1-p}+v r^{1+p / 1-p} \quad \text { if } \quad r>0 \text { is large enough; }
$$

(b) there exists $L=\lim _{r \rightarrow \infty} r^{-(2 / 1-p)} u(r)$. Moreover, $L=c_{p, N}$ or $L=0$.

Proof. (a) Let $x_{0}$ be a point in $\mathbb{R}^{N}$ such that $u\left(x_{0}\right)<+\infty$, and let $\mu>2 u\left(x_{0}\right)$. Consider the problem

$$
u=u\left(\left|x-x_{0}\right|\right), \quad-\Delta u+u^{p}=0 ; u\left(x_{0}\right)=\mu, \quad \nabla u\left(x_{0}\right)=0
$$

and denote by $u_{\mu}(r)$ its solution. Notice that $u_{\mu}(r)$ is a radial solution shifted with respect to the origin. Using the results in [4], it follows that for large $r>0$

$$
u_{\mu}(r) \leqq c_{p, N} r^{2 / 1-p}+v r^{1+p / 1-p} .
$$

Assume that the result is not true. Then we may suppose that $u(R)>u_{\mu}(R)$ for some $R>0$, so that by the maximum principle, $u(r) \geqq u_{\mu}(r)$ in $B_{R}$, whence in particular $u\left(x_{0}\right) \geqq u_{\mu}\left(x_{0}\right)=2 u\left(x_{0}\right)$, a contradiction.

(b) Let $z(r)=k r^{2 / 1-p}$. Ther, for large $r, z(r)$ is a subsolution of (1.4) (respectively a supersolution) if $k>c_{p, N}$ (respectively $k<c_{p, N}$ ). By part (a), $\lim \sup _{r \rightarrow \infty} r^{-(2 / 1-p)} u(r)<+\infty$. If

$$
0 \leqq L_{1} \equiv \liminf _{r \rightarrow \infty} r^{-(2 / 1-p)} u(r)<\underset{r \rightarrow \infty}{\limsup } r^{-(2 / 1-p)} u(r) \equiv L_{2} \leqq c_{p, N}<+\infty,
$$


then we would obtain a contradiction by comparing $u(r)$ and $z(r)$ with $c<c_{p, N}$ as in Lemma 2.1. Therefore $L_{1}=L_{2}=L$. To compute $L$, we notice that, since

$$
r^{1-N}\left(r^{N-1} u^{\prime}(r)\right)^{\prime}=u^{p}-f(r)
$$

we have for $r \geqq R_{0}$

$$
u^{\prime}(r)=r^{-1 N} \int_{R_{0}}^{r} s^{N-1}\left(u^{p}(s)-f(s)\right) d s+r^{1-N} u^{\prime}\left(R_{0}\right)
$$

Using then L'Hôpital's rule:

$$
\lim _{r \rightarrow \infty} r^{-(1+p / 1-p)} u^{\prime}(r)=\left(\frac{1+p}{1-p}+N-1\right)^{-1} \lim _{r \rightarrow \infty}\left(r^{-(2 / 1-p)} u(r)\right)^{p}=\left(\frac{1+p}{1-p}+N-1\right)^{-1} L^{p}
$$

and therefore

$$
L=\lim _{r \rightarrow \infty} r^{-(2 / 1-p)} u(r)=\lim _{r \rightarrow \infty}\left(\frac{2}{1-p} r^{1+p / 1-p}\right)^{-1} u^{\prime}(r)=c_{p, N}^{1-p} L^{p},
$$

whence the result

We now show the existence of a unique minimal solution.

LemMa 2.9. There exists a unique solution $u_{0}(r)$ of the problem

$$
-u^{\prime \prime}-\frac{N-1}{r} u^{\prime}+u^{p}=f, \quad f(r)=o\left(r^{2 p / 1-p}\right) \quad \text { as } \quad r \rightarrow \infty
$$

such that $u_{0}(r)=o\left(r^{2 / 1-p}\right)$ as $r \rightarrow \infty$.

Proof. Existence is trivial if $f(r) \equiv 0$. As to the general case, consider for simplicity the situation where $N \geqq 3$. Let us show first that (1.4) has now a supersolution $w(r)$ such that $w(r) \approx \varepsilon r^{2 / 1-p}$ for $r \rightarrow \infty$, where $\varepsilon>0$ is small enough. To this end, we take

$$
w(r)=\varepsilon r^{2 / 1-p}+E_{N} * f_{1}+M,
$$

where $f_{1}$ is as in (2.12), and $M$ is a positive constant to be selected presently. We have

$$
-w^{\prime \prime}-\frac{N-1}{r} w^{\prime}+w^{p} \geqq\left(\varepsilon^{p}-\varepsilon c_{p, N}^{p-1}\right) r^{2 p / 1-p}+f_{1},
$$

where we have used the fact that $E_{N} * f_{1} \geqq 0$. Take now $\varepsilon>0$ such that $\varepsilon^{p}-\varepsilon c_{p, N}^{p-1}>0$. Since $f(r)=o\left(r^{2 p / 1-p}\right)$ as $r \rightarrow \infty$ and $f_{1} \geqq 0$ almost everywhere, it follows that there exists $R_{0}>0$ such that, for $r>R_{0}$,

$$
-w^{\prime \prime}-\frac{N-1}{r} w^{\prime}+w^{p} \geqq f(r) .
$$

On the other hand, we may always assume $f=f_{1}$ when $r<R_{0}$, so that taking $M>0$ large enough we also obtain $-w^{\prime \prime}-(N-1 / r) w^{\prime}+w^{p} \geqq 0$ there. 
Once a supersolution has been constructed, existence follows by approximation techniques as in [3, Thm 1]. Uniqueness is shown in [3, Thm. 5]. Finally, since $0 \leqq u_{0} \leqq \varepsilon r^{2 / 1-p}$ as $r \rightarrow \infty$, we deduce from Lemma 2.8(b) that $u_{0}(r)=o\left(r^{2 / 1-p}\right)$ as $r \rightarrow \infty$. The cases $N=1,2$ can be dealt with as in Remark 2.6.

We next show that for fixed $k$, the solution given in (2.15) is unique. To this end, we prove

Lemma 2.10. Let $u(r)$ be a solution of (1.4) such that (2.15) holds. Then for $\varepsilon>0$ small enough, there holds

$$
u(r)-c_{p, N} r^{2 / 1-p}-w_{R}(r)-k \sigma(r)=O\left(r^{\gamma}\right) \quad \text { as } \quad r \rightarrow \infty,
$$

where

$$
\gamma=\max \left\{\frac{2}{1-p}-2\left|\alpha_{+}\right|+\varepsilon, \frac{2}{1-p}-\left|\alpha_{-}\right|+\varepsilon\right\}
$$

and $\alpha_{+}, \alpha_{-}$are given in (2.11).

Proof. Let us define

$$
\mu(r)=u(r)-c_{p, N} r^{2 / 1-p}-w_{R}(r)-k \sigma(r) .
$$

Proceeding as in the proof of Lemma 2.4, and taking into account that $\mu \ll \sigma$ and $w_{R} \ll r^{2 / 1-p}$ as $r \rightarrow \infty$, we obtain

$$
-\mu^{\prime \prime}-\frac{N-1}{r} \mu^{\prime}+p\left(c_{p, N} r^{2 / 1-p}+w_{R}\right)^{p-1} \mu=O\left(\frac{|\sigma|^{2}}{r^{(2 / 1-p)(2-p)}}\right) \quad \text { as } \quad r \rightarrow \infty .
$$

Arguing as in Lemma 2.3, we then get

$$
\mu(r)=a_{1} \psi_{+}(r)+a_{2} \psi_{-}(r)+S(r) \quad \text { as } \quad r \rightarrow \infty,
$$

where $S(r)$ is the quantity corresponding to the term $O\left(r^{-(2 / 1-p)(2-p)}|\sigma|^{2}\right)$ via variation of constants formula, whereas $\psi_{+}, \psi_{-}$are two linearly independent solutions of the homogeneous version of (2.18). We then have $S(r) \ll$ $r^{(2 / 1-p)-2 \mid \alpha_{+}+\varepsilon}$ as $r \rightarrow \infty$, for some small enough $\varepsilon>0$. Taking into account (2.10), and the fact that $\mu(r)=o\left(\psi_{+}(r)\right)$ as $r \rightarrow \infty$, we obtain that $\mu(r)=a_{2} \psi_{-}(r)+S(r)$, and the result follows

Lemma 2.10 will be used in deriving

LEMMA 2.11. For any fixed real $k$, there exists at most one solution $u(r)$ of (1.4) satisfying (2.15).

Proof. Let us argue by contradiction. Assume then that there exist two such solutions $u_{1}$ and $u_{2}$. Then $z=u_{1}-u_{2}$ satisfies

$$
-z^{\prime \prime}-\frac{N-1}{r} z^{\prime}+V(r) z=0,
$$

where

$$
V(r)=\frac{u_{1}^{p}-u_{2}^{p}}{u_{1}-u_{2}}=u_{1}^{p}\left[\frac{1-\left(u_{2} / u_{1}\right)^{p}}{1-\left(u_{2} / u_{1}\right)}\right]
$$


so that, by (2.15)

$$
V(r)=\frac{p c_{p, N}^{p-1}}{r^{2}}\left[1+O\left(\frac{w_{R}(r)+\sigma(r)+r^{\gamma}}{r^{2 / 1-p}}\right)\right] \text { as } \quad r \rightarrow \infty .
$$

By (2.19) and (2.20), it follows that

$$
z(r)=\alpha_{1} K_{1}(r)+\alpha_{2} K_{2}(r),
$$

where $K_{1}(r)$ and $K_{2}(r)$ satisfy asymptotic conditions (2.10). Since by Lemma 2.10 $z(r)=o\left(r^{(2 / 1-p)-\left|\alpha_{+}\right|+\varepsilon}\right)$ as $r \rightarrow \infty$, we deduce that $z(r)=\alpha_{2} K_{2}(r)$, so that $z(r) \rightarrow 0$ as $r \rightarrow \infty$. As $|z|$ is subharmonic in the whole space (by Kato's inequality), we deduce that $z \equiv 0$.

To conclude the proof of Theorem 1.1, it only remains to show that every nontrivial, nonminimal solution $u(r)$ of (1.4) is in the form (2.15). Indeed, by Lemma 2.8(b), one then has that $u(r) \approx c_{p, N} r^{2 / 1-p}$ as $r \rightarrow \infty$. Let $w_{R}(r)$ be as in Lemma 2.1, and set

$$
\xi(r)=u(r)-c_{p, N} r^{2 / 1-p}-w_{R}(r) .
$$

Then $\xi(r)$ satisfies

$$
-\xi^{\prime \prime}-\frac{N-1}{r} \xi^{\prime}+\frac{p \xi}{(g(r))^{1-p}}=O\left(\frac{\xi^{2}}{(g(r))^{2-p}}\right) \quad \text { as } \quad r \rightarrow \infty,
$$

where $g(r)=c_{p, N} r^{2 / 1-p}+w_{R}(r)$. We have thus obtained a nonhomogeneous version of (2.7), with an extra term which goes to zero as $r \rightarrow \infty$ faster than $1 / r^{2}$. Arguing as in Lemma 2.10, we obtain that

$$
\xi(r)=k \tilde{\psi}_{+}(r) \text { for some real } k,
$$

where

$$
r^{(2 / 1-p)-\left|\alpha_{+}\right|-\varepsilon} \ll \tilde{\psi}_{+}(r) \ll r^{(2 / 1-p)-\left|\alpha_{+}\right|+\varepsilon} \text { as } r \rightarrow \infty,
$$

and the proof is concluded.

We next analyse the problem corresponding to a particular choice of $f(r)$. To this end, we need the following technical result:

Lemma 2.12. Let $f(r)$ be such that $f(r)=o\left(r^{2 p / 1-p}\right)$ as $r \rightarrow \infty$ and

$$
\int^{\infty} t^{-(1+p / 1-p)} f(t) d t<+\infty .
$$

Then, if $w_{R}(r)$ is the function considered in Lemma 2.1, $g(s)=s^{-(2 / 1-p)}\left|w_{R}(s)\right|$ satisfies

$$
\int^{\infty} \frac{g(s)}{s} d s<+\infty .
$$

Proof. For simplicity, we shall drop the subscript $R$ in what follows. From (1.7) and (2.2) we deduce that, for large enough $r, w(r)$ is a solution of

$$
-w^{\prime \prime}-\frac{N-1}{r} w^{\prime}+\frac{p c_{p, N}^{p-1}}{r^{2}} w=f(r)+O\left(r^{-(2+(2 / 1-p))} w^{2}\right) .
$$


Recalling the arguments in the proof of Lemma 2.3, using the variation of constants formula in (2.23), and taking into account that $\left|\alpha_{-}\right|>\left|\alpha_{+}\right|$, we get that, for some $r_{0}$ large enough and any $r>r_{0}$,

$$
\begin{aligned}
|w(r)| \leqq & a r^{(2 / 1-p)-|\alpha+|}+C r^{(2 / 1-p)-|\alpha+|} \\
& \times \int_{r_{0}}^{r} s^{-((1+p / 1-p)-\mid \alpha+1)}\left[f(s)+O\left(s^{-(2+(2 / 1-p))}(w(s))^{2}\right)\right] d s
\end{aligned}
$$

for some positive constants $a$ and $C$. Since, for any given $\varepsilon>0,|w(s)| \leqq \varepsilon s^{2 / 1-p}$ if $s \geqq r_{0}$ with $r_{0}$ sufficiently large, we then arrive at

$$
\begin{aligned}
|w(r)| \leqq & \gamma_{\varepsilon} r^{(2 / 1-p)-\left|\alpha_{+}\right|}+C r^{(2 / 1-p)-\mid \alpha+1} \int_{r_{0}}^{r} s^{-((1+p / 1-p)-\mid \alpha+1)} f(s) d s \\
& +\varepsilon r^{(2 / 1-p)-\left|\alpha_{+}\right|} \int_{r_{0}}^{r} s^{-\left((2 / 1-p)+1-\mid \alpha_{+}\right)}|w(s)| d s
\end{aligned}
$$

for some $\gamma_{\varepsilon}>0$. Now set

$$
\phi(r)=\int_{r_{0}}^{r} s^{-\left((2 / 1-p)+1-\mid \alpha_{+}\right)}|w(s)| d s, \quad \Psi(r)=\int_{r_{0}}^{r} s^{-\left((1+p / 1-p)-\mid \alpha_{+}\right)} f(s) d s .
$$

We shall denote henceforth by $C$ a generic constant, depending only on $p$ and $N$. A routine computation in (2.24) yields

$$
\left(r^{-\varepsilon} \phi(r)\right)^{\prime} \leqq \gamma_{\varepsilon} r^{-(1+\varepsilon)}+C r^{-(1+\varepsilon)} \Psi(r)
$$

whence, by integration,

$$
\phi(r) \leqq a_{2} r^{\varepsilon}+C r^{\varepsilon} \int_{r_{0}}^{r} s^{-(1+\varepsilon)} \Psi(s) d s
$$

for some $a_{2}=a_{2}(\varepsilon)>0$. Substituting this in (2.24), we obtain

$$
\begin{aligned}
|w(r)| \leqq \gamma_{\varepsilon} r^{(2 / 1-p)-\left|\alpha_{+}\right|}+C r^{(2 / 1-p)-\left|\alpha_{+}\right|} \Psi(r) & \\
& +\varepsilon r^{(2 / 1-p)-\left|\alpha_{+}\right|}\left(a_{2} r^{\varepsilon}+C r^{\varepsilon} \int_{r_{0}}^{r} s^{-(1+\varepsilon)} \Psi(s) d s\right) .
\end{aligned}
$$

We shall use (2.25) to show that (2.22) holds. In doing so, one is led to estimate the various terms arising there. The essential point is to bound $\int_{r_{0}}^{\infty} s^{-\left(1+\mid \alpha_{+}\right)} \Psi(s) d s$. To this end, we compute

$$
\begin{aligned}
\int_{r_{0}}^{\infty} s^{-(1+|\alpha+|)} \Psi(s) d s= & \int_{r_{0}}^{\infty} s^{-\left(1+\mid \alpha_{+}\right)}\left[\int_{r_{1}}^{s} \xi^{-\left((1+p / 1-p)-\mid \alpha_{+}\right)} f(\xi) d \xi\right] d s \\
= & \int_{r_{0}}^{\infty} s^{-\left((1+p / 1-p)-\mid \alpha_{+}\right)} f(s) \\
& \times\left[\int_{s}^{\infty} r^{-\left(1+\left|\alpha_{+}\right|\right)} d r\right] d s \leqq C \int_{r_{0}}^{\infty} s^{-(1+p / 1-p)} f(s) d s
\end{aligned}
$$

and this last integral is bounded by assumption. Once this has been done, the remaining terms are dealt with in a straightforward way.

An immediate consequence of Theorem 1.1 and Lemma 2.12 is 
Proposition 2.13. Assume that $f(r)=o\left(r^{2 p / 1-p}\right)$ as $r \rightarrow \infty$, and (2.21) holds. Then the set of nonminimal solutions of (1.4) consists of a monoparametric family of functions $u_{k}(r),-\infty<k<+\infty$, such that, for any fixed $k$,

$$
u_{k}(r)=c_{p, N} r^{2 / 1-p}+w_{R}(r)+k r^{(2 / 1-p)-\left|\alpha_{+}\right|}+o\left(r^{(2 / 1-p)-\left|\alpha_{+}\right|}\right) \text {as } r \rightarrow \infty .
$$

Proof. It suffices to see that, in equation (2.7a), we have

$$
p\left(c_{p, N} r^{2 / 1-p}+w(r)\right)^{p-1}=\frac{p c_{p, N}^{p-1}}{r^{2}}\left(1+r^{-(2 / 1-p)} w(r)+O\left(r^{-2(2 / 1-p)} w^{2}\right)\right) \quad \text { as } \quad r \rightarrow \infty .
$$

We then make the substitution $r=e^{y}$, and use [1, Theorem 8, Chapter 2], to conclude.

\subsection{Small perturbations in the subcritical case}

In what follows, we shall assume that $f(r)$ in (1.4) satisfies $f(r)=c r^{2 p / 1-p}+$ $g(r)$, with $0<c<c_{*}$ (cf. (1.13)), and $g(r)=o\left(r^{2 p / 1-p}\right)$ as $r \rightarrow \infty$. We want to show Theorem 1.2 here. Since the arguments required are very much alike to those already employed in the proof of Theorem 1.1, we shall just sketch their main lines, and refer in each case to the related results in Section 2.1 for the details. Let $\xi_{R}(r)$ be as in (1.6), and consider the problem

$$
\begin{gathered}
w^{\prime \prime}-\frac{N-1}{r} w^{\prime}+\left[\left(c_{2} r^{2 / 1-p}+w\right)^{p}-c_{2}^{p} r^{2 p / 1-p}\right] \xi_{R}(r)=\xi_{R}(r) g(r), \\
w(0)=w^{\prime}(0)=0,
\end{gathered}
$$

where $c_{2}$ is the largest root of (1.14). Then there exists a unique solution $\tilde{w}_{R}(r)$ of (2.26). Moreover

$$
\bar{w}_{R}(r) \leqq 0 \text { for } r>0 \text { and } w_{R}(r)=o\left(r^{2 / 1-p}\right) \text { as } r \rightarrow \infty .
$$

The proof of (2.27) is quite analogous to that of Lemma 2.1. Let us define now $\bar{\sigma}(r)$ as the solution (if any) of the following problem:

$$
\begin{gathered}
-\sigma^{\prime \prime}-\frac{N-1}{r} \sigma^{\prime}+p\left(c_{2} r^{2 / 1-p}+\bar{w}_{R}(r)\right)^{p-1} \xi_{R} \sigma=0 \text { for } r>0, \\
\sigma(0)=1, \quad \sigma^{\prime}(0)=0 .
\end{gathered}
$$

Arguing as in Lemma 2.3, we obtain that there exists a unique solution $\tilde{\sigma}(r)$ of (2.28). Moreover, $\bar{\sigma}(r)>0$ for any $r, \bar{\sigma}(r) \rightarrow \infty$ as $r \rightarrow \infty$ and, for $\varepsilon>0$ small enough,

$$
r^{(2 / 1-p)-\left|\beta_{+}\right|-\varepsilon} \ll \bar{\sigma}(r) \ll r^{(2 / 1-p)-\left|\beta_{+}\right|+\varepsilon} \quad \text { as } \quad r \rightarrow \infty,
$$

where $\beta_{+}$is the largest root of

$$
\lambda^{2}+\beta_{p, N} \lambda-\left(p c^{p-1}-c_{p, N}^{p-1}\right)=0 .
$$

As a next step, we show that for any real $k$, there exists a solution $u_{k}(r)$ of (1.4) under our current hypothesis, such that

$$
u_{k}(r)=c_{2} r^{2 / 1-p}+\bar{w}_{R}(r)+k \bar{\sigma}(r)+o(\bar{\sigma}(r)) \text { as } r \rightarrow \infty,
$$


where $R>0$ is large enough. As in Section 2.1, this is done by a standard use of sub- and supersolutions. More precisely, we take as a subsolution $(\varphi-M)_{+}$, with $M>0$ large enough, and

$$
\varphi(r)=c_{2} r^{2 / 1-p}+\bar{w}_{R}(r)+k \bar{\sigma}(r)-\bar{v}(r),
$$

where $\bar{v}(r)=\delta r^{(2 / 1-p)-\left\{\beta_{+} \mid-\varepsilon\right.}, \varepsilon>0$ is small enough, and $\varepsilon, \delta$ are taken such that

$$
-\bar{v}^{\prime \prime}-\frac{N-1}{r} \bar{v}^{\prime}+\frac{p c_{2}^{p-1}}{r^{2}} \bar{v}=r^{(2 p / 1-p)-|\beta+|-\varepsilon}
$$

As to the supersolution, we assume for simplicity $N \geqq 3$ and try $(\psi+M)$, where again $M>0$ is large enough and

$$
\psi(r)=c_{2} r^{2 / 1-p}+\bar{w}_{R}(r)+k \sigma(r)+\lambda(r)+v(r) .
$$

Here, as in Lemma 2.5, $\lambda(r)=E_{N} * f_{1}, f_{1}$ being as in (2.14). Uniqueness of solutions satisfying (2.30) is obtained in two steps. We show first that, if $u(r)$ is a solution of (1.4) satisfying (2.30), then

$$
u(r)=c_{2} r^{2 / 1-p}+\bar{w}_{R}(r)+k \sigma(r)+O\left(r^{\chi}\right) \text { as } r \rightarrow \infty,
$$

where

$$
\chi=\max \left\{\frac{2}{1-p}-2\left|\beta_{+}\right|+\varepsilon, \frac{2}{1-p}-\left|\beta_{-}\right|+\varepsilon\right\} .
$$

Here $\beta_{+}, \beta_{-}$are the roots of (2.29), and $\varepsilon>0$ is small enough, so that $\chi<0$. The corresponding result in the previous section is Lemma 2.10, whose proof is easily adapted to the case under consideration. We then argue by contradiction, and assume that there exist two solutions $u_{1}, u_{2}$ of (1.4) satisfying (2.30). Setting $z=u_{1}-u_{2}$, we slightly modify the argument in Lemma 2.11 to show that $z(r)=b K(r)$, for some constant $b$, where $K(r) \rightarrow 0$ as $r \rightarrow \infty$. Since $|z|$ is subharmonic in the whole space by Kato's inequality, we deduce that $z \equiv 0$.

We now turn our attention to the existence of minimal solutions. As in the previous paragraph, we shall need the following auxiliary tool:

Let $u(r)$ be a solution of (1.4) under our current assumptions. Then

$$
u(r) \leqq c_{p, N} r^{2 / 1-p}+k r^{1+p / 1-p}
$$

for some real $k$ and sufficiently large $r$.

The proof of (2.23) follows from the maximum principle as in Lemma 2.8(a). Estimate (2.32) is then used to obtain that, if $u(r)$ is as before,

$$
\text { There exists } L=\lim _{r \rightarrow \infty} r^{-(2 / 1-p)} u(r), \quad \text { and } L=c_{1} \text { or } c_{2} \text {, }
$$

where $c_{1}, c_{2}$ are the roots of (1.14). The proof of (2.33) is a minor variant of that of part (b) in Lemma 2.8.

We are then in a position to show that there exists a minimal solution $u_{1}(r)$ of (1.4), and $u_{1}(r) \approx c_{1} r^{2 / 1-p}$ as $r \rightarrow \infty$.

This follows by means of a standard sub- and supersolution argument. As a subsolution, we just take $\varphi(r) \equiv 0$, and as a supersolution we consider, when 
$N \geqq 3$.

$$
\psi(r)=\left(c_{1}+\varepsilon\right) r^{2 / 1-p}+E_{N} * g_{1}+M
$$

with $\varepsilon>0$ small enough and $g_{1}$ obtained by replacing $f$ by $g$ in (2.14). Once again, the case $N \leqq 2$ is dealt with as in Remark 2.6.

It only remains to show that the minimal solution thus constructed is unique. To this end, we first show that if $u(r)$ solves (1.4) and $u(r) \approx c_{1} r^{2 / 1-p}$ as $r \rightarrow \infty$, then

$$
u(r)=c_{1} r^{2 / 1-p}+\tilde{w}_{R}(r)+O\left(r^{-(2 p / 1-p)-N}\right) \text { as } \quad r \rightarrow \infty,
$$

where $\bar{w}_{R}(r)$ satisfies

$$
\begin{gathered}
-w^{\prime \prime}-\frac{N-1}{r} w^{\prime}+\left(\left(c_{1} r^{2 / 1-p}+w\right)^{p}-c_{1}^{p} r^{2 p / 1-p}\right) \xi_{R}=\xi_{R} g, \\
w(0)=w^{\prime}(0)=0 .
\end{gathered}
$$

The proof of (2.34) is quite analogous to that of (2.31) (cf. Lemma 2.10). Once (2.34) has been obtained, uniqueness follows by a contradiction argument. We just look to the equation satisfied by the difference of two possible solutions, $z=u_{1}-u_{2}$. As in Lemma 2.11, application of (2.34) and Kato's inequality then yield $z \equiv 0$.

Finally, the fact that every solution of $(1.4),(1.2)$ is described in $(1.15),(1.16)$ follows now as in Section 2.1.

We conclude this section by stating without proof a result which is the analogue of Proposition 2.13.

Proposition 2.14. Assume that $f(r)=c r^{2 p / 1-p}+g(r)$, where $g(r)=o\left(r^{2 p / 1-p}\right)$ as $r \rightarrow \infty$ and $\int^{\infty} t^{-(1+p / 1-p)} g(t) d t<+\infty$. Then the set of nonminimal solutions of (1.4) consists of a monoparametric family of functions $u(r ; k),-\infty<k<+\infty$, such that, for any fixed $k$,

$$
u(r ; k)=c_{2} r^{2 / 1-p}+\bar{w}_{R}(r)+k r^{(2 / 1-p)-\left|\beta_{+}\right|}+o\left(r^{(2 / 1-p)-\left|\beta_{+}\right|}\right) \quad \text { as } \quad r \rightarrow \infty,
$$

where $\bar{w}_{R}(r)$ solves (2.26) with $R>0$ large enough, and $\beta_{+}$is the largest root of (2.29).

\section{The proof of Theorem 1.3}

Throughout this section we shall assume that $f(r)=c_{*} r^{2 p / 1-p}+h(r)$, where $h(r)=o\left(r^{2 p / 1-p}\right)$ as $r \rightarrow \infty$ and $c_{*}$ is given in (1.13).

\subsection{The case $h(r) \equiv 0$}

We shall consider first the situation where $f(r)=c_{*} r^{2 p / 1-p}$. Due to the homogeneity of the problem, we then may use a phase space approach. As in [4], we perform the change of variables

$$
u(r)=r^{2 / 1-p} v(y), \quad r=e^{y},
$$


to obtain the autonomous system

$$
\left\{\begin{array}{l}
\dot{v}=w \\
\dot{w}=\left(v^{p}-c^{-p}\right)-\beta_{p, N} w-c_{p, N}^{p-1}(v-\bar{c}),
\end{array}\right.
$$

where $\bar{c}$ is the unique root of (1.14) under our current hypothesis. When looking to the corresponding phase portrait, it is readily seen that there are only two trajectories corresponding to global solutions of $(1.4)$ : the equilibrium $(\bar{c}, 0)$, which corresponds to the unique solution of (1.4) with $u(0)=u^{\prime}(0)=0$, and a single trajectory $\gamma$ approaching $(\bar{c}, 0)$ from the region where $v>\bar{c}$ and $w<0$, which corresponds to solutions of (1.4) with $u(0)>0, u^{\prime}(0)=0$. The behaviour of this last curve near $(\bar{c}, 0)$ is now described.

Lemma 3.1. Let $(v(y), w(y))$ be on the trajectory y referred to above. Then

$$
\begin{gathered}
\lim _{y \rightarrow \infty} \frac{w(y)}{(v(y)-\bar{c})^{2}}=-\frac{p(1-p)}{2(\bar{c})^{2-p} \beta_{p, N}} \equiv-A_{1} ; \\
\lim _{y \rightarrow \infty} \frac{1}{(v(y)-\bar{c})^{3}}\left[w(y)+A_{1}(v(y)-\bar{c})^{2}\right]=A_{2},
\end{gathered}
$$

where

$$
A_{2}=\frac{1}{\beta_{p, N}}\left[\frac{2 p(1-p)(2-p)}{3(\bar{c})^{3-p}}-\frac{p^{2}(1-p)^{2}}{2(\bar{c})^{3-p} \beta_{p, N}^{2}}\right]
$$

There exists

$$
\lim _{y \rightarrow \infty} \frac{1}{(v(y)-\bar{c})^{4}}\left[w(y)+A_{1}(v(y)-\bar{c})^{2}-A_{2}(v(y)-\bar{c})^{3}\right]=A_{3},
$$

where $A_{3}$ is a real constant depending on $p$ and $N$.

Proof. Let us begin by that of (3.3). Consider the curve

$$
\sum \equiv \sum_{k}=\left\{(v, w): w=-k(v-\bar{c})^{2}, k>0\right\}
$$

and define $s(v)=\dot{w} / \dot{v}$. A straightforward calculation shows that $s(v)>d w / d v$ along $\Sigma$ (respectively $s(v)<d w / d v$ along $\Sigma$ ), provided that $p(p-1) / 2(\bar{c})^{2-p}+$ $\beta_{p, N} k<0$ (respectively $>0$ ). Therefore an appropriate choice of $k$ yields that there exists $L=\lim _{y \rightarrow \infty} w(y) /(v(y)-\bar{c})^{2}$, and since the eigenvalues of the linearization of (3.1) near $(\bar{c}, 0)$ are $-\beta_{p, N}$ and 0 , we also have that $\lim _{y \rightarrow \infty} w(y) /(v(y)-\bar{c})=0$. Clearly, $-\infty \leqq L \leqq 0$, and it is readily seen that $L>-\infty$. Indeed, if $L=-\infty$, using L'Hôpital's rule we would get

$$
\lim _{y \rightarrow \infty} \frac{w(y)}{(v(y)-\bar{c})}=\lim _{y \rightarrow \infty} \frac{\dot{w}(y)}{\dot{v}(y)}=\lim _{y \rightarrow \infty} \frac{p(p-1)}{2(\bar{c})^{2-p}} \cdot \frac{(v-\bar{c})^{2}}{w}-\beta_{p, N}=-\beta_{p, N},
$$


a contradiction. To compute $L$, we again use L'Hôpital's rule to get

$$
L=\lim _{y \rightarrow \infty}\left[\frac{p(p-1)}{2(\bar{c})^{2-p}}-\beta_{p, N} \frac{w(y)}{(v(y)-\bar{c})^{2}}\right]\left[2\left[\frac{w(y)}{(v(y)-\bar{c})}\right]\right]^{-1}
$$

and since the denominator vanishes, it follows that (3.3) holds. The proofs of (3.4), (3.5) consist in minor adaptations of that of (3.3). As to (3.4), we replace our initial choice of $\sum$ by

$$
\sum_{1, k}=\left\{(v, w): w=A_{1}(v-\bar{c})^{2}+k(v-\bar{c})^{3}\right\}
$$

and obtain $A_{2}$ by using L'Hôpital's rule to estimate the various terms arising from the left-hand side of (3.4). To get (3.5), we start instead with curves

$$
\sum_{2, k}=\left\{(v, w): w=A_{1}(v-\bar{c})^{2}-A_{2}(v-\bar{c})^{3}+k(v-\bar{c})^{4}\right\} .
$$

We now have

Proposition 3.2. Let $f(r)=c_{*} r^{2 p / 1-p}$. Then the set of solutions of (1.4), (1.2) consists of a function $u_{0}(r)=\bar{c} r^{2 / 1-p}$ (the minimal solution), and a monoparametric family of functions $u_{0}(r ; k),-\infty<k<+\infty$, such that

$$
u_{0}(r ; k)=u_{a}(r)+\frac{k r^{2 / 1-p}}{(\ln r)^{2}}+O\left(\frac{r^{2 / 1-p}}{(\ln r)^{3-\varepsilon}}\right) \text { as } r \rightarrow \infty,
$$

where $\varepsilon$ is any number between 0 and 1 , and

$$
\begin{gathered}
u_{a}(r)=\bar{c} r^{2 / 1-p}+a_{1} \frac{r^{2 / 1-p}}{\ln r}+a_{2} r^{2 / 1-p} \frac{\ln (\ln r)}{(\ln r)^{2}}, \\
a_{1}=\frac{1}{A_{1}}=\frac{2 \bar{c}^{2-p} \beta_{p, N}}{p(1-p)}, \\
a_{2}=\frac{a_{1}^{3}}{\beta_{p, N}}\left[\frac{2 p(1-p)(2-p)}{3(\bar{c})^{3-p}}-\frac{p^{2}(1-p)^{2}}{2(\bar{c})^{3-p} \beta_{p, N}^{2}}\right] .
\end{gathered}
$$

Proof. Let $(v(y), w(y))$ be on the trajectory $\gamma$ described at the beginning of this section. Then by Lemma 2.1,

$$
\begin{aligned}
\begin{array}{l}
w(y)=-A_{1}(v(y)-\bar{c})^{2}+A_{2}(v(y)-\bar{c})^{3}+A_{3}(v(y)-\bar{c})^{4} \\
+o\left((v(y)-\bar{c})^{4}\right) \text { as } y \rightarrow \infty .
\end{array}
\end{aligned}
$$

Now set $\psi(y)=v(y)-\bar{c}$. Then by (3.6), $d \psi / d y \leqq-\left(A_{1} / 2\right) \psi^{2}$ for large enough $y$, so that there exists a real $k_{1}$ such that $\psi(y) \leqq k_{1} / y$ for large enough $y$. On the other hand, by the choice of $\psi,(3.7)$ can be recast as

$$
\frac{d}{d y}\left(\frac{1}{\psi}\right)=A_{1}-A_{2} \psi-A_{3} \psi^{2}+o\left(\psi^{2}\right)
$$

whence, after integration in $y$, we get

$$
\psi(y)=\frac{1}{A_{1} y}+O\left(\frac{\ln y}{y^{2}}\right) \quad \text { as } \quad y \rightarrow \infty .
$$


Use of (3.9) in (3.8) yields after integration

$$
\psi(y)=\left[C+A_{1} y-\frac{A_{2}}{A_{1}} \ln y+O\left(\frac{1}{y^{1-\varepsilon}}\right)\right]^{-1}
$$

for some real constant $C$, and arbitrary $\varepsilon \in(0,1)$. Back to the original variables $(r, u(r))$ and expanding the quantity within the braces, we get

$$
\begin{aligned}
u_{m}(r)= & \bar{c} r^{2 / 1-p}+\frac{1}{A_{1}} \frac{r^{2 / 1-p}}{\ln r}+\frac{A_{2}}{A_{1}^{3}} \frac{r^{2 / 1-p} \ln (\ln r)}{(\ln r)^{2}} \\
& -\frac{C}{A_{1}^{2}} \frac{r^{2 / 1-p}}{(\ln r)^{2}}+O\left(\frac{r^{2 / 1-p}}{(\ln r)^{3-\varepsilon}}\right) \text { as } r \rightarrow \infty
\end{aligned}
$$

where, by scaling, $C \equiv c(1)-A_{1} \ln \left(m^{1-p / 2}\right)$.

\subsection{An existence result}

In this paragraph we shall assume that for some $\varepsilon>0$

$$
f(r)=c_{*} r^{2 p / 1-p}+h(r), \quad \text { where } \quad h(r)=O\left(\frac{r^{2 p / 1-p}}{(\ln r)^{2+\varepsilon}}\right) \quad \text { as } \quad r \rightarrow \infty .
$$

Let $u_{a}(r)$ be the function given in (3.5), and consider the problem

$$
\begin{gathered}
-w^{\prime \prime}-\frac{N-1}{r} w^{\prime}+u_{a}(r)^{p}\left[\left(1+\frac{w(r)}{u_{a}(r)}\right)^{p}-1\right] \xi_{R}(r)=\xi_{R}(r) h(r), \quad r>0, \\
w(0)=w^{\prime}(0)=0,
\end{gathered}
$$

where for fixed $R>0, \xi_{R}(r)$ is as (1.6). Then there holds

LEMMA 3.3. For $R>0$ large enough, there exists a unique global solution of (3.11), $w_{R}^{*}(r)$. Moreover, for $\varepsilon>0$ small enough

$$
\begin{gathered}
\varepsilon \frac{r^{2 / 1-p}}{\ln r} \geqq w_{R}^{*}(r) \geqq-\varepsilon \frac{r^{2 / 1-p}}{\ln r} \text { if } r>0 \text { is large, } \\
\lim _{r \rightarrow \infty} w_{R}^{*}(r)\left(\frac{r^{2 / 1-p}}{\ln r}\right)^{-1}=0 .
\end{gathered}
$$

Proof. Let us check the second inequality in (3.12). We shall show that for $\varepsilon>0$ small enough, $\zeta(r)=-\varepsilon \frac{r^{2 / 1-p}}{\ln r}$ is a supersolution of (3.11a). To this end, we remark that, after some routine computations, we have for large $r>0$

$$
\begin{aligned}
-\zeta^{\prime \prime}-\frac{N-1}{r} \zeta^{\prime} & +u_{a}(r)\left[\left(1+\frac{\zeta(r)}{u_{a}(r)}\right)^{p}-1\right]-f(r) \equiv L(\zeta)-f \\
& \geqq \frac{\varepsilon r^{2 p / 1-p}}{(\ln r)^{2}}\left(-\beta_{p, N}+p a_{1}(\bar{c})^{p-2}+\frac{\varepsilon p(p-1)}{2 \bar{c}^{2}}-p^{2} a_{1}(\bar{c})^{p-2}\right)+\ldots
\end{aligned}
$$

where $a_{1}$ is given in (3.6b). We thus obtain that there exists $\varepsilon_{0}$ such that $L(\zeta)-f>0$ if $0<\varepsilon<\varepsilon_{0}$ (respectively $L(\zeta)-f<0$ if $\varepsilon>\varepsilon_{0}$ ). The proof of the first inequality in (3.12) is similar, and will be omitted. As to (3.13) one readily sees that for $\varepsilon>0$ small enough, $\bar{w}(r)=\varepsilon r^{2 / 1-p}(\ln r)^{-1}$ is a subsolution of (3.11a) 
for large $r$. We then may argue as in Lemma $2.8(\mathrm{~b})$, to deduce that $L=$ $\lim _{r \rightarrow \infty} w_{R}^{*}(r)\left[r^{2 / 1-p}(\ln r)^{-1}\right]^{-1}$ must exist, and moreover, $L=-a_{1}$ or $L=0$. Taking into account (3.12) the result follows.

We next show existence of solutions referred to in the statement of Theorem 1.3(a). To begin with, we obtain the following lemma:

Lemma 3.4. Assume that (3.10) holds. Then there exists a solution $u^{*}(r)$ of (1.4) such that

$$
u^{*}(r)=\bar{c} r^{2 / 1-p}+o\left(\frac{r^{2 / 1-p}}{\ln r}\right) \quad \text { as } \quad r \rightarrow \infty .
$$

Proof. Suppose for simplicity that $N \geqq 3$. It is then readily seen that

$$
\varphi_{1}(r)=\bar{c} r^{2 / 1-p}+\varepsilon \frac{r^{2 / 1-p}}{\ln r}+E_{N} * f_{1}+M, \text { where } \varepsilon>0
$$

is small enough, $M>0$ is sufficiently large, and $f_{1}$ is as in (2.12), is a supersolution of (1.4);

$$
\varphi_{2}(r)=\left(\bar{c} r^{2 / 1-p}-\frac{\varepsilon r^{2 / 1-p}}{\ln r}-M\right)_{+} \text {is a subsolution of }
$$

(1.4) for $\varepsilon>0$ small enough and $M>0$ sufficiently large.

The conclusion follows then from (3.14) and standard approximation and suband supersolution methods, together with asymptotic arguments similar to those in Lemma 2.1.

Lemma 3.5. Assume that (3.10) holds. Then for any real $k$ there exists a unique solution of $(1.4), u^{*}(r ; k)$, such that

$$
u^{*}(r ; k)=u_{a}(r)+w_{R}^{*}(r)+k \frac{r^{2 / 1-p}}{(\ln r)^{2}}+O\left(\frac{r^{2 / 1-p}}{(\ln r)^{3-\varepsilon}}\right) \quad \text { as } \quad r \rightarrow \infty,
$$

where $u_{a}(r)$ and $w_{R}^{*}(r)$ are given in (3.6) and Lemma 3.3 respectively, and $\varepsilon$ is any number between 0 and 1 .

Proof. It follows from standard techniques as soon as suitable sub- and supersolutions are available. Assume for convenience $N \geqq 3$. Taking into account Proposition 3.2, we have that

$\Psi_{1}(r)=\left(u_{0}(r ; k)+w_{R}^{*}(r)-\frac{r^{2 / 1-p}}{(\ln r)^{2+\varepsilon}}-M\right)_{+}$, where $\varepsilon>0$ is sufficiently small, $M>0$ is large enough, and $u_{0}(r ; k)$ is given in Proposition 3.2, is a subsolution of $(1.4)$;

$\Psi_{2}(r)=\left(u_{0}(r ; k)+w_{R}^{*}(r)+\frac{r^{2 / 1-p}}{(\ln r)^{2+\varepsilon}}-E_{N} * f_{1}+M\right)$,

where $M>0$ is large enough and $f_{1}$ is given in (2.12), is a supersolution of (1.4). 
To conclude with the proof of part (a) in Theorem 1.3, it remains to show uniqueness, and this is done by a suitable adaptation of the corresponding arguments in Section 2 (cf. Lemmata 2.9-2.11 and remarks following this last Lemma).

\subsection{A nonexistence result}

We now assume that, in (1.4)

$$
\begin{aligned}
& f(r)=c_{*} r^{2 p / 1-p}+h(r), \text { where for large enough } r, \\
& h(r)>0 \text { and } h(r) \geqq \frac{b r^{2 p / 1-p}}{(\ln r)^{\gamma}} \text { for some } b>0 \text { and } \\
& 0<\gamma<2 .
\end{aligned}
$$

We perform again the change of variables (3.1), to obtain now, instead of (3.2), the nonautonomous system

$$
\left\{\begin{array}{l}
\dot{v}=w, \\
\dot{w}=\left(v^{p}-(\bar{c})^{p}\right)-\beta_{p, N} w-c_{p, N}^{p-1}(v-\bar{c})-H(y),
\end{array}\right.
$$

where $H(y)=e^{-(2 p y / 1-p)} h\left(e^{y}\right)$. An analysis of the direction field associated to (3.17) reveals that the only way to approach $(\bar{c}, 0)$ is through region $A$, where

$$
A=\left\{(v, w): v>\bar{c}, w \leqq \frac{1}{\beta_{p, N}}\left(v^{p}-(\bar{c})^{p}\right)-c_{p, N}^{p-1}(v-\bar{c}) \leqq 0\right\} .
$$

On the other hand, along the curve $w=-k(u-\bar{c})^{2}, k<p(1-p) / 2(\bar{c})^{2-p} \beta_{p, N}$, the field is directed downwards in the homogeneous case, and so does for (3.17) (at least in some neighbourhood of $(\bar{c}, 0)$ ), since $H(y)>0$ for large $y$. Thus, starting from some $\left(v_{0}, w_{0}\right)$ close enough to $(\bar{c}, 0)$ with $v_{0}=v\left(y_{0}\right), w_{0}<-k\left(v_{0}-\right.$ $\bar{c})^{2}$, we have that for $y>y_{0}$

$$
\dot{v}(y)=w(y)<-k(v(y)-\bar{c})^{2},
$$

whence

$$
v(y)-\bar{c} \leqq \frac{k_{1}}{y} \text { for large enough } y .
$$

We now go back to the second equation in (3.17), which can be written in the form

$$
\dot{w}=-\frac{p(1-p)}{2(\bar{c})^{2-p} \beta_{p, N}}(v-\bar{c})^{2}-\beta_{p, N} w+O\left((v-\bar{c})^{3}\right)+H(y)
$$

where, by assumption,

$$
H(y)>\frac{1}{y^{\gamma}} \text { if } y \text { is large enough. }
$$

In what follows we shall denote by $k_{i}(i=1,2,3, \ldots)$ several generic constants depending only on $y_{0}, p$ and $n$. Taking into account (3.19) and (3.21), (3.20) 
yields $w+\beta_{p, N} w \leqq-\left(k_{2} / y^{\gamma}\right)$ whence, by (3.18),

$$
w(y)<-\frac{k_{3}}{y^{\gamma}} \text { for } y \text { large enough. }
$$

Putting together (3.19) and (3.22), we obtain $\left(v(y)-\bar{c} /|w(y)|^{1 / \gamma}\right)<k_{4}$ or

$$
|w(y)|>k_{4}(v-\bar{c})^{\gamma}, \quad \gamma<2
$$

We have thus obtained a refinement to (3.18). In particular, if $\gamma<1$, integrating (3.23) in $y$ yields

$$
(v(y)-\bar{c})^{1-\gamma}<\alpha-\beta y \quad \text { for some real } \alpha \text { and } \beta,
$$

whence $v(y)=\bar{c}$ at some finite $y$, whereas $|w(y)|>\left(k_{3} / y^{\gamma}\right)$ there. This shows that our trajectory has to leave region $A$, thus losing any possibility of eventually approaching $(\bar{c}, 0)$ and the nonexistence result is obtained.

Assume now $1<\gamma<2$. Then integrating (3.23) in $y$ gives $(v(y)-\bar{c})^{\gamma-1}<$ $\left(k_{5} / y\right)$ whence $\left(v(y)-\bar{c} /|w(y)|^{1 / \gamma}\right)>k_{6}$ and therefore

$$
|w(y)|>k_{7}(v-\bar{c})^{\gamma^{2}-\gamma} \text {. }
$$

Notice that, since $\gamma<2, \gamma^{2}-\gamma<\gamma$. We can then establish an iterative argument, obtaining at the $n$th step (cf. (3.23), (3.24))

$$
\dot{v}<-M_{n}(v-\bar{c})^{\alpha_{n}}, \quad|w(y)|>N_{n}(v-\bar{c})^{\gamma \alpha_{n}-\gamma}
$$

for some $M_{n}>0$ and $N_{n}>0$, so that, assuming $\alpha_{n} \neq 1$ for any $n$, we have

$$
\alpha_{n}=\gamma^{n}-\left(\gamma^{n-1}+\ldots+\gamma\right)=\frac{(\gamma-2) \gamma^{n}+\gamma}{\gamma-1} .
$$

Since $1<\gamma<2, \alpha_{n} \rightarrow-\infty$ as $n \rightarrow \infty$, and after a finite number of steps we are reduced to the case $\gamma<1$. Finally, if $\alpha_{n}=1$ for some $n$, we just notice that then

$$
\dot{v}<-M_{n}(v-\bar{c})<-M_{n}(v-\bar{c})^{1+\varepsilon} \text { for some } \varepsilon>0
$$

and reduce ourselves to the previously considered cases. This concludes the proof.

\section{References}

1 R. Bellman. Stability theory of differential equations (New York: Dover, 1953).

2 H. Brezis. Semilinear equations in $\mathbb{R}^{N}$ without conditions at infinity. Appl. Math. Optim. 12 (1984), 271-282.

3 T. Gallouët and J. M. Morel. The equation $-\Delta u+|u|^{\alpha-1} u=f$ for $0 \leqq \alpha \leqq 1 . J$. Nonlinear Anal. 11 (1987), 893-912.

4 M. A. Herrero and J. J. L. Velázquez. On the dynamics of a semilinear heat equation with strong absorption. Comm. Partial Differential Equations 14 (1989), 1653-1715.

5 M. Murata. Structure of positive solutions to $(-\Delta+V) u=0$ in $\left[\mathbb{R}^{N}\right.$. Duke Math J. 53 (1986), $869-943$. 